\title{
Using Health Information Technology to Enhance a Culture of Safety
}

\section{Aziz HA*}

College of Arts and Sciences, Qatar University, Qatar

\section{Introduction}

It was astonishing to learn that as many as 98,000 Americans die annually in hospitals due to preventable medical errors. This number is expected to increase as many of the medical care occur in ambulatory settings outside hospitals. To Err is Human: Building a Safer Health System by the Institute of Medicine (IOM), had raised sufficient interest to a degree that patient safety is now recognized as a national health care issue. Therefore, a focus should be aimed at reducing medical errors and system failures by collecting and analyzing data to gain sufficient information, and bring attention to what the healthcare system is lacking [1].

Despite the emphasis on patient safety, errors occur. Patients may receive the wrong medication or fall getting out of bed, both causing serious safety events. These events arise as a result of the untimely combination of high-risk actions (human error or lack of compensatory actions), and high-risk conditions (environments or situations that increase the probability for error) [2]. Most of the significant reasons for morbidity and mortality are due to medication errors that are confirmed and stated by the IOM report. They account for around 130 outpatient deaths and about 854 inpatient deaths. In 2007, IOM published another report on medication safety calling for preventing medication errors. This report highlighted the main issue in reducing medication based errors, monitoring and reporting errors, improving patient-physician communication, providing decision-support tools for physicians, and standardizing the labeling of medication.

\section{Culture of safety}

A culture of safety is an idealistic environment where all mistakes caused by medical errors do not happen due to the presence of safetyconscious values. Thompson defined the term as "A safety culture exists within an organization [when] each individual employee, regardless of their position, assumes an active role in error prevention and that role is supported by the organization" [3]. A culture of safety is a mechanism that focuses on the safety and effective care of the patient. The term represents an organization that has adopted measures to decrease actual or potential adverse events. These measures may include transparency, employee involvement in practice decisions, emphasizing on safe behaviors and a reporting system that's non punitive, and focused on system failures [4]. Several studies showed a strong link between a safety culture, error reporting and decreasing adverse events $[5,6]$. Most of the studies on patient safety promote data collection, reporting, obtaining leadership and reducing blames [7].

\section{Advocating patient safety}

Ensuring patient safety is one of the most vital and challenging jobs in healthcare. World Health Organization (WHO) established the Information Technology for Patient Safety Expert Working Group to examine the role of Information Technology (IT) in improving patient safety in healthcare. The Federal Government, by the Patient Safety and Quality Improvement Act encouraged patient safety culture and created patient safety organizations (PSOs) that collect, combine and analyze private, confidential data, and information stated by health care providers. Analysis of patient safety events allows PSOs to recognize problems, and reduce or eliminate hazards and risks that affect patient safety [2]. Furthermore, most of the clinicians and health care providers worry from reporting patient safety events fearing these reports could be used against them. The Act solved these kinds of fears by providing legal regulations to protect the confidentiality of the reported information by the providers. Moreover, this Act restricted the use of this information in illegal and administrative purposes; penalties for violation of protection were included in this Act. The Act aimed to establish a network of patient safety databases to offer integrated, interactive and management that was evidence-based for PSOs, entities and health care providers [8].

An example of implementing a culture of safety is the Military Health System (MHS). MHS has been successful in improving the quality of healthcare and enhancing the safety of patients during the delivery of the health services [9]. The goal of improving patient safety by MHS was achieved through:

- Collecting good information and data for the patients that help in making clinical decisions

- Electronic reporting of medical results for fast retrieval of data and sharing with other physicians

- Computerized order entry system to reduce the illegibility problems

- Clinical support decision systems were used for drug prescription

At the government level, the Food and Drug Administration (FDA) regulates food, drugs, medical devices and biological products for human use. The effectiveness of the FDA's drug safety monitoring procedures was called into question after several approved drugs were shown to have serious side-effects. In September 2006, an IOM report commissioned by the FDA found that its drug safety system was limited by inadequate funding, insufficient regulatory authority, and a lack of oversight by experts free of pharmaceutical industry ties. In its concluding report, the IOM recommended healthcare professionals to strive to create a culture of safety, and to report medical errors without fear of retaliation.

The Joint Commission (JC) works continuously to improve the safety and quality of patient care through the provision of healthcare accreditation services to support performance improvement in facilities. Public reporting of preventable medical errors forces hospitals to account medical error numbers accurately and improve the quality

*Corresponding author: Hassan A Aziz, PhD MLS(ASCP) $\mathrm{cm}$, Associate Dean for Academic Affairs, College of Arts and Sciences, Qatar University, P.O. Box: 2713, Doha, Qatar, Tel: 0097444034783; E-mail: Hassan.Aziz@qu.edu.qa

Received August 03, 2016; Accepted August 17, 2016; Published August 27 , 2016

Citation: Aziz HA (2016) Using Health Information Technology to Enhance a Culture of Safety. J Biosens Bioelectron 7: 218. doi: 10.4172/2155-6210.1000218

Copyright: (C) 2016 Aziz HA. This is an open-access article distributed under the terms of the Creative Commons Attribution License, which permits unrestricted use, distribution, and reproduction in any medium, provided the original author and source are credited. 
of care being delivered [10]. Non-governmental PSOs are also involved in patient safety. Organizations like Leapfrog Group, Health Grades, National Patient Safety Foundation, American Hospital Association and US Pharmacopeia are collecting and defining the best practices from all over the country.

\section{Medication errors}

Medication error is defined as an inappropriate use of medication that will harm the patient. This error results from professional malpractice, healthcare products misuse, and inappropriate procedures or system usage. Furthermore, medication error can result from medication prescribing, drug labeling, packaging, distribution, and order communication [11].

Medication errors happen due to many reasons and cause adverse drug event (ADE) in some cases. These medication errors, most of the time, occur when there is complex dosing, and those drugs given in certain areas such as emergency, intensive care unit, and diagnostic departments are strongly linked with great chance to get ADE [12]. Researchers showed that there are 15 different types of medication errors including: missed use, wrong dose, wrong drug, wrong choice, wrong time, known allergy, wrong technique, wrong frequency, drugdrug interaction, wrong administration route, failure to act, extra dose, preparation errors insufficient monitoring and other types. Out of 130 medication errors caused by clinicians, the most common error was due to wrong dose, followed by wrong choice of medication. On the other hand, among 126 administration errors caused by nurses, the most common error was due to wrong dose, followed by wrong technique and wrong drug [10]. The most common type of medication errors results in death of the patients such as wrong dose (40.9\%), wrong medication (16\%) and wrong route of drug administration (9.5\%) [13]. Another study showed that the worst type of ADE is strongly associated with drugs for cardiovascular, central nervous system and neoplastic diseases.

\section{The five rights}

An error can occur at any of the following steps of prescribing, transcribing, dispensing, administering drugs, and monitoring patient response that comprises the complex multistep process in medication administration. Although many errors arise at the prescribing stage, some are captured by pharmacists, nurses, or other staff; yet, administration errors account for $26 \%$ to $32 \%$ of total medication errors [14]. Medication errors in healthcare settings can have severe consequences for patients who are given the wrong medication or dose, or who are given medication incorrectly. Regarded as the gold standard, and one of the fundamentals for safe medication practices, is the "five rights" concept [15]. It comprises: 1) Right patient where patient's identity is verified against the medication order ensuring that the correct patient is receiving the medication. In addition, verification by matching the patient's hospital wrist band against those on the medication order, 2) Right medication step involves verifying the medication before administration in which medication is confirmed three times: once when the medication is removed from the drug storage area, once before placing the medication on a patient's tray or medication cup, and again before giving the medication to the patient, 3) Right time to administer medications to the patient's at a specified time ordered by the physician, 4) Right dose is confirmed against the written medication order before being given while preparing the medication, when placing the medication in the patient's medication cup, and again before giving the medication to the patient, and 5) Right Route to ensure the must the correct route of the medication injection or intravenously through a vein - for delivering the ordered medication as advised by the physician [16].

Despite the fact that the "five rights" are practiced by the health care provider, problems and errors still occur [17]. The reason for that is lack of clear guidance to health care providers to ensure the safety of the drug; for example, when the pharmacist finds difficulty determining the right patient when the name of the patient or his ID information is not clear, or the physician's handwriting is illegible. Figure 1 lists a number of factors that facilitate the medical provider's failure to achieve the goal of the "five rights".

\section{Technologies and improvement of patient safety}

Patient safety technology is defined as using practical and applied methods to deliver healthcare to the patients. Errors could be reduced using information technology by preventing medication errors and adverse events, enhancing effective response after adverse event, and reporting and providing feedback about the adverse events [18]. Patient safety technology can be categorized into four main groups [19]:

- Support the direct care of the patients

- Support the documentation

- Support the needs of patients and families

- Support the staffs who care for patients

Technology can be delivered at the bedside of the patient. Bedside monitoring of blood pressure, hemodynamics, cardiac rhythm and oxygenation are important methods to monitor patients in acute and critical situation. These types of bedside monitoring techniques provide quick, suitable and effective response, and intervention [20,21].

Bar coded medication administration (BCMA) systems in another example for use of technology in today's healthcare. The aim of designing BCMAs is to promote patient safety through incorporating the medication administration record of the patient, following and confirming the "five rights" methods, and guaranteeing that health care providers follow the equipment and medication guidelines [11].
1- Insufficient staff
2- Medical services are not well designed
3- Illegible handwriting
4- Removing zeroes (e.g., .3 instead of 0.3 ) will lead to misinterpretation in prescribing the dose of the drug
5- Unclear drug labeling
6- Lack of double check system for certain drugs

Figure 1: Factors affecting the five rights. 
Recent studies confirmed that BCMA systems can reduce medication and administration errors [12]. Linear and 2D bar coded medication administration can be used by a nurse to verify the identity of the patient and the correct match with the prescribed medication [22].

Automated medication dispensing devises are becoming widely used and they are usually located beside nurses' counters. These devices communicate with pharmacy computers and dispense medications by the pharmacy. Also, the use of robots in dispensing medication is seen at many medical centers. Smart intravenous infusion pumps can be programed to deliver the correct amount of IV drugs and are associated with drugs libraries and alerts. As an added benefit, the pumps are connected to the internet and can transmit data so events can be captured and investigated. Radio frequency identification (RFID) tags have been used in commercial stores for a while. They are now used in healthcare. The tags are read by a scanner with databases to identify objects with high accuracy. RFID are used to track patients and inventory.

Technological tools are not limited to medical facilities. Home electronic medication management system can be placed in the homes of elderly and disabled patients. These systems are connected to a medical provider and a pharmacy via the internet to monitor compliance with medications and to adjust medication dosage when necessary.

In conclusion, safe medical performance depends on the best available resources. To achieve a culture of safety, healthcare should reach a balance between doing the right things and producing desirable outcomes in reliable and efficient way. Combining both direct us how to "do the right things right".

\section{References}

1. Cooper JB (2001) Current research on patient safety in the United States. National Patient Safety Foundation. pp: 62.

2. Huckvale C, Car J, Akiyama M, Jaafar S, Khoja T, et al. (2010) Information technology for patient safety. The international journal of healthcare improvement 19: i25-i33.

3. Thompson, Elizabeth M (2011) Defining a culture of safety. OR nurse journa 5: 3 .

4. Braithwaite J, Westbrook MT, Travaglia JF, Hughes C (2010) Cultural and associated enablers of, and barriers to, adverse incident reporting. Qual Sa Health Care 19: 229-233.
5. Estabrooks CA, Tourangeau AE, Humphrey CK, Hesketh KL, Giovannetti $P$, et al. (2002) Measuring the hospital practice environment: a Canadian context. Res Nurs Health 25: 256-268.

6. Moody RF, Pesut DJ, Harrington CF (2006) Creating safety culture on nursing units: Human performance and organizational system factors that make difference. Journal of Patient Safety 2: 198-206.

7. Piotrowski MM, Hinshaw DB (2002) The safety checklist program: creating a culture of safety in intensive care units. Jt Comm J Qual Improv 28: 306-315.

8. http://www.ahrq.gov/qual/psoact.htm

9. Tang PC Chair (2003) Key capabilities of an electronic health record system. A report of the Committee on Data Standards for Patient Safety. Institute of Medicine, National Academy Press, Washington, DC.

10. Leape LL, Bates DW, Cullen DJ, Cooper J, Demonaco HJ, et al. (1995) Systems analysis of adverse drug events. JAMA 274: 35-43.

11. Koppel R, Wetterneck T, Telles JL, Karsh BT (2008) Workarounds to barcode medication administration systems: their occurrences, causes, and threats to patient safety. J Am Med Inform Assoc 15: 408-423.

12. Poon EG, Cina JL, Churchill W, Gandhi TK (2006) Medication dispensing errors and potential adverse drug events before and after implementing bar code technology in the pharmacy. Ann Intern Med 145: 426-34.

13. Phillips J, Beam S, Brinker A, Holquist C, Honig P, et al. (2001) Retrospective analysis of mortalities associated with medication errors. Am J Health Syst Pharm 58: 1835-1841.

14. Anderson P (2010) Medication errors: Don't let them happen to you. American Nurse Today.

15. 2004, ISMP Medication Safety Alert! 5 Nurse Advise-ERR. Institute for Safe Medication Practices.

16. Otto E (2010) 5 Rights of Medication safety. Living Strong Health.

17. Grissinger M (2010) The Five Rights A Destination Without a Map.

18. Kuperman GJ, Teich JM, Bates DW (1997) Improving care with computerized alerts and reminders. Assoc for Health Serv Res 14: 224-225.

19. Henneman EA (2010) Patient Safety and Technology. AACN Advanced Critical Care 20: 128-132.

20. Smith HA, Lee SH, O'Neill PA, Connolly MJ (2000) The combination of bedside swallowing assessment and oxygen saturation monitoring of swallowing in acute stroke: a safe and humane screening tool. Age Ageing 29: 495-499.

21. Collet JP, Cuisset T, Rangé G (2012) Bedside monitoring to adjust antiplatelet therapy for coronary stenting. N Engl J Med 367: 2100-2109.

22. Shah DK, Lavery S, Doyle LW, Wong C, McDougall P, et al. (2006) Use of 2-channel bedside electroencephalogram monitoring in term-born encephalopathic infants related to cerebral injury defined by magnetic resonance imaging. Pediatrics 118: 47-55 